\title{
Non-Metallic Modified Thermal Barrier Coatings
}

\author{
A.O. Badalyan \\ A.I. Alikhanyan National Scientific Laboratory, Alikhaniyan Bros. Str., \\ Yerevan, 375036, Armenia
}

Emai: anush.badalyan@yerphi.am

Received 7 April 2021

\begin{abstract}
The radiation resistance and optical properties of zinc and zirconium silicate doped with cerium ion obtained by the hydrothermal microwave method are investigated. To study the radiation resistance of materials, the samples were irradiated with electrons energy of $4 \mathrm{MeV}$ and a dose of $10^{16} \mathrm{el} / \mathrm{cm}^{2}$. X-ray diffraction (XRD) technique was used to characterize the phase composition of materials. To study the optical properties of materials, photoluminescence spectra were studied. Using the comparative section method, the thermal conductivity of the materials was measured. The results show that thermal barrier coatings made of cerium-doped zinc and zirconium silicate can be excellent candidates for use in space crafts and not only.
\end{abstract}

https://doi.org/10.52853/18291171-2021.14.2-85

Keywords: hydrothermal-microwave synthesis, zirconium silicate, zinc silicate, thermal barrier coatings, thermal conductivity, photoluminescence

\section{Introduction}

The main tendencies of modern spacecraft and instrumentation are the increase in the active life of spacecraft (SC) to 10-15 years, the not tightness of their design, and the widespread use of new polymer composite materials. Therefore, it is relevant to use physical modeling methods for a comprehensive study of the behavior of spacecraft materials under conditions of forced ground action of the main factors of outer space- deep vacuum, fluxes of high-energy charged particles, electromagnetic radiation from the Sun, thermal cycling, etc. [1-3].

Many compositions are known for TBC's of the class "solar reflectors" such as enamel, modified oxide of zirconium, zinc, and others, which are not stable under conditions of exposure to space factors, do not provide antistatic properties, since they are deep dielectrics. This leads to violation of stability in the operation of radio-electronic equipment, to malfunctions and failures of onboard systems and, thereby, to a decrease in the active life of space equipment and with long periods of orbital flight, a sufficiently large number of defects and centers of absorption, luminescence are formed [4-6]. Therefore, the development of methods for increasing the photoand radiation resistance of TBC pigments is an urgent problem.

One of the promising methods to solve this problem can be the modification of pigments with various oxide and rare-earth based nanopowders, since nanoparticles, having large specific surface area, promote relaxation of defects formed upon irradiation with high-energy particles.

Thus, studies to assess the radiation resistance of TBC powders, to determine the effect of modification conditions, the type and concentration of nanopowders on the optical properties, stability to the action of various types of radiation are of scientific and practical interest.

The purpose of this work is to investigate the radiation resistance and optical properties of zinc and zirconium silicate doped with cerium by X-ray diffraction analysis methods and optical spectroscopy (photoluminescence). Calculation of the coefficients of thermal conductivity of the samples is performed as well. 


\section{Materials and techniques}

The synthesis of hydrosilicate zirconium was carried out in a microwave oven brand "CE1073AR" of "Samsung" company, in an open glass flask equipped with a reflux condenser and stirrer. Synthesis conditions were: microwave frequency - $2.45 \mathrm{GHz}$, microwave power - 600 Watts. Concentrations of initial aqueous solutions of $\mathrm{ZrSO}_{4}$ and $\mathrm{Na}_{2} \mathrm{O} \cdot \mathrm{SiO}_{2}$ were $0.5 \mathrm{~mol} / \mathrm{l}$. The synthesis was carried out by reaction of zirconium sulfate and sodium silicate solutions, maintaining the $\mathrm{pH}$ of the medium at 7.0 [7].

Aqueous solutions of zinc chloride $\left(\mathrm{ZnCl}_{2}, 99.5 \%\right)$ and $\mathrm{Na}_{2} \mathrm{SiO}_{3}$ with the concentration of 1.0 mol/l were mixed and irradiated in a MS-6 "VOLTA" microwave oven ( $2.45 \mathrm{GHz}$ frequency) for 1.5-3.5 hours. The maximum temperature during the synthesis was $\sim 240{ }^{\circ} \mathrm{C}$. Cerium (III) nitrate hexahydrate $\left(\mathrm{CeH}_{12} \mathrm{~N}_{3} \mathrm{O}_{15}\right)(99.0 \%)$ was added to solutions for cooking 5\% wt. pigments $\mathrm{Ce}-$ $\mathrm{Zn}_{2} \mathrm{SiO}_{4}$. This concentration was chosen to ensure alloying of the material, and not to form the second phase. After microwave irradiation, the precipitates were filtered and washed with warm deionized water to remove $\mathrm{NaCl}$ by-products and then was dried at $110{ }^{\circ} \mathrm{C}$ [8].

Irradiation of samples was carried out at A. Alikhanyan National Laboratory using a linear electron accelerator with energy of $4 \mathrm{MeV}$, and irradiation dose $10^{16} \mathrm{el} / \mathrm{cm}^{2}$. The average electron flux density was maintained at approximately $10^{12} \mathrm{~cm}^{-2} \mathrm{~s}^{-1}$. The temperature of the samples during irradiation did not exceed $25{ }^{\circ} \mathrm{C}$.

For complete removal of bound water, the samples of zinc orthosilicate $\mathrm{Ce} / \mathrm{Zn}_{2} \mathrm{SiO}_{4}\left(1050{ }^{0} \mathrm{C}\right)$ and zirconium $\mathrm{Ce} / \mathrm{ZrSiO}_{4}\left(1200{ }^{\circ} \mathrm{C}\right)$ were heat treated. The X-ray phase analysis of the samples was carried out by the powder method. X-ray powder diffraction (XRD) is a rapid analytical technique primarily used for phase identification of a crystalline material and can provide information on unit cell dimensions. The analyzed material is finely ground, homogenized, and the average bulk composition is determined.

For optical studies, spectroscopic methods were used, which are based on the interaction of electromagnetic radiation with a material. The LUMEN experimental facility is designed for spectral measurements with a high spectral resolution, wide temperature range. A $1 \mathrm{~kW}$ DKSEL 1000-5 xenon ultra-high-pressure lamp was used as a light source. The registration system made it possible to measure luminescence spectra at various spectral points with selective photoexcitation up to $\sim 5 \mathrm{eV}$.

As already mentioned, high-quality coatings should have low thermal conductivity, for this, the thermal conductivity coefficients of our samples were measured. In this work, we used the comparative section method. The comparative cut-bar technique is a steady-state method for the measurement of thermal conductivity of solids.

The measurements were performed at room temperature. Fiberglass with thermal conductivity of $0.3 \mathrm{~W} /(\mathrm{m} \cdot \mathrm{K})$ was used as a standard material.

\section{Results and discussions}

As it is known, under the high-energy radiation, radiation defects and electronic excitations arise in the TBC [7-11]. The conditions for irradiation of thermoregulatory silicate materials, for example, with electrons, were selected taking into account the possibility of their stay in the Earth's radiation belt for more than 10 years. Since silicate compounds have a complex structure, it is not always possible to substantiate the system of defects responsible for the formation of certain radiation color centers. During exposure to ionizing radiation, traps present in the material and responsible for the formation of color centers filled with electrons and holes, which leads to an increase in the optical density of silicate materials. 
The mechanism of action of different parts of the radiation spectrum on the material environment is completely different, which is determined by the atomic-molecular structure of the substance. When powders are irradiated with TBC, two types of color centers are formed:

- radiation defects caused by nonstoichiometry of the crystal structure and on intrinsic point defects of the surface;

- defects formed during irradiation in the volume of the crystal lattice.

The concentration of defects of the first type largely depends on the specific surface area and grain size of the powders. As the specific surface area increases, the concentration of defects usually increases or changes along a curve with a maximum.

When light quanta and ionizing radiation act on powders (defects of the second type), electron-hole pairs are formed, after which their separation is possible. The holes formed move to the surface, where they interact with the adsorbed $\mathrm{O}_{2}, \mathrm{H}_{2} \mathrm{O}$ molecules and impurities. Free electrons can increase electrical conductivity and reduce surface potential barriers between coating grains. It follows that decompositions are characteristic both under the action of light quanta or radiation with energies greater than the energy gap of the powder, and under the action of electrons with an energy lower than the forbidden gap of the powder (not sufficient for the formation of Frenkel pairs).

It is known that the luminescence spectra of some simple oxides $\left(\mathrm{BeO}, \mathrm{Al}_{2} \mathrm{O}_{3}\right)$, may contain several bands of intrinsic luminescence, but in complex crystals, such as TBC, there can be several sublattices and structural fragments of different types respectively and defects formed as a result of heat treatment, radiation and the presence of impurity ions [12]. Doping with cerium helps to obtain highly crystalline nanoscale structure as shown in our previous work [8]. It also stabilizes crystalline structure, due to the difference between the electric field of the $\mathrm{Ce}_{3}{ }^{+} / \mathrm{Ce}_{4}{ }^{+}$and $\mathrm{Zn}_{2}{ }^{+}, \mathrm{Zr}_{2}{ }^{+}$ cations. This difference can lead to a decrease in the total energy of the lattice, which will make it more stable under irradiation with high-energy electrons [8].

The phase composition of the TBC was investigated using X-ray diffraction analysis (XRD) which follows that the samples obtained have a crystalline structure. Fig. 1 shows the X-ray diffraction patterns of the $\mathrm{Ce}-\mathrm{ZrSiO}_{4}$ and $\mathrm{Ce}-\mathrm{Zn}_{2} \mathrm{SiO}_{4}$ samples. The $\mathrm{Ce}-\mathrm{ZrSiO}_{4}$ sample has diffraction peaks of a tetragonal structure with lattice parameters $a=b=0.65989 \mathrm{~nm}, \mathrm{c}=0.59857$ $\mathrm{nm}$. The average grain size is $19.1 \mathrm{~nm}$ (from the Scherrer equation) (Fig. 1a).

The $\mathrm{Ce}-\mathrm{Zn}_{2} \mathrm{SiO}_{4}$ sample exhibits broad diffraction peaks of the rhombohedral structure of $\alpha$ $\mathrm{Zn}_{2} \mathrm{SiO}_{4}$ with the space group $\mathrm{R}-3$ and the cell constant $\mathrm{a}=\mathrm{b}=1.395 \mathrm{~nm}, \mathrm{c}=0.9312 \mathrm{~nm}$. The average grain size is $20.2 \mathrm{~nm}$ (Fig. 1b).

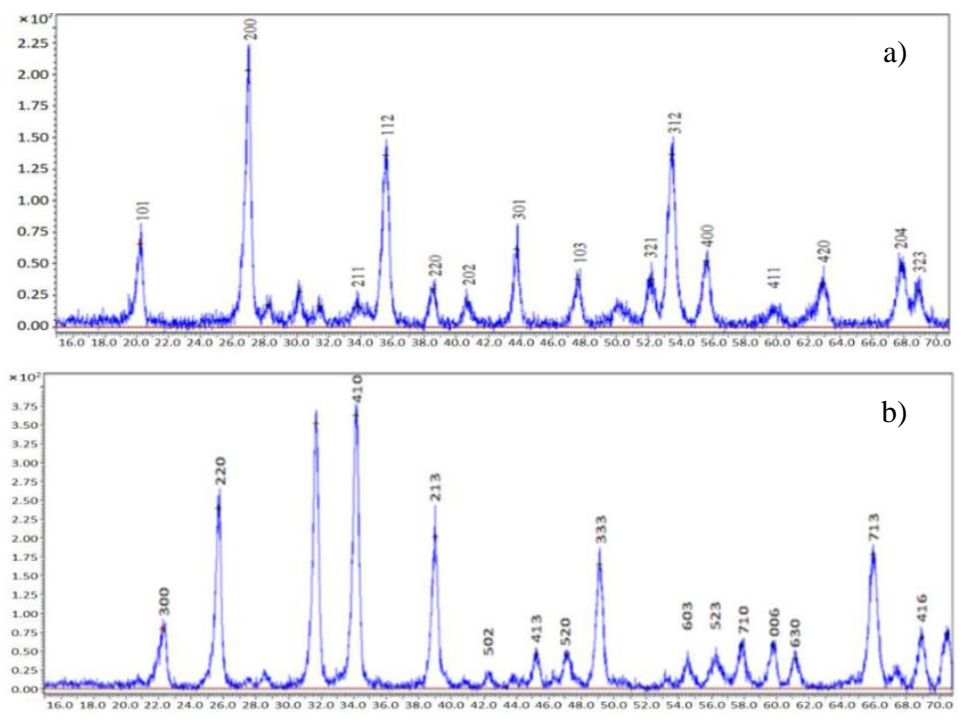

Fig. 1. X-ray diffraction patterns of $\mathrm{Ce}-\mathrm{ZrSiO}_{4}$ (a) and $\mathrm{Ce}-\mathrm{Zn}_{2} \mathrm{SiO}_{4}$ (b) synthesized by microwave-assisted. 
To evaluate in more detail the TBC radiation resistance, some optical properties of the samples were investigated before and after electron irradiation. Irradiation point defects serve as traps for electrons and holes and become color centers. Electrons and holes fill these centers, which leads to an increase in the optical density of silicates. Optical density depends on the radiation dose rate, as well as on the isothermal rate of annealing of color centers.

Fig. 2 shows the photoluminescence spectra of the samples before and after irradiation with electrons with energy of $4 \mathrm{MeV}$ and a dose of $10^{16} \mathrm{e} / \mathrm{cm}^{2}$.
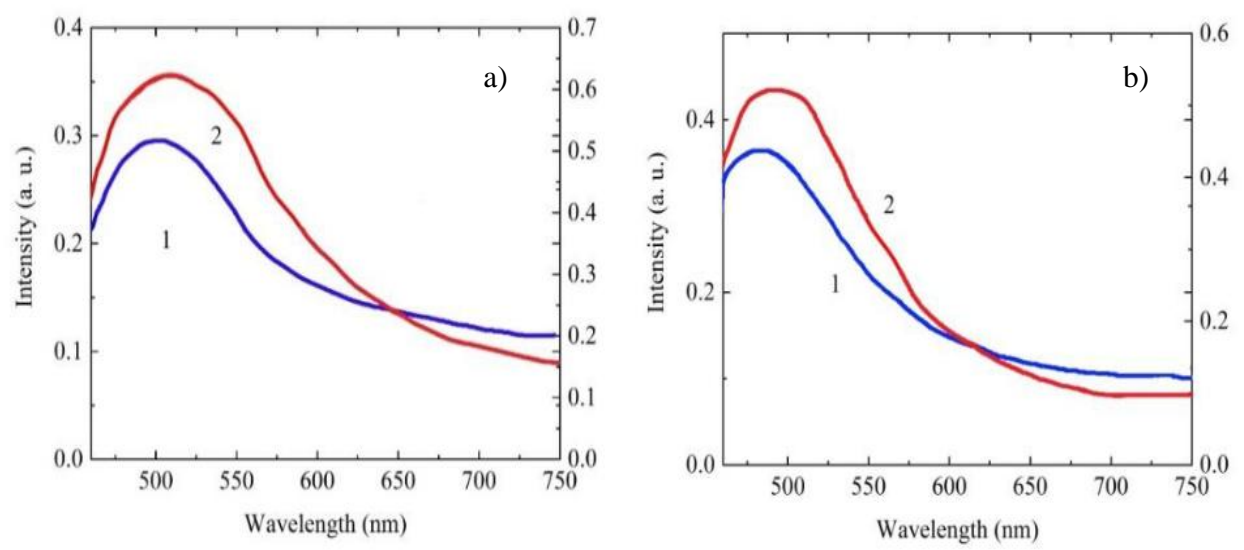

Fig. 2. Photoluminescence spectra of samples a) $\mathrm{ZrSiO}_{4}\left(\mathrm{Ce}_{2} \mathrm{O}_{3}-5 \%\right)$, b) $\mathrm{Zn}_{2} \mathrm{SiO}_{4}\left(\mathrm{Ce}_{2} \mathrm{O}_{3}-5 \%\right), \mathrm{T}=300 \mathrm{~K}$, $\mathrm{E}_{\mathrm{exc}}=3.06 \mathrm{eV}$ (1-before irradiation, 2-after irradiation with electrons with an energy of $4 \mathrm{MeV}$ and a dose of $10^{16} \mathrm{el} / \mathrm{cm}^{2}$ )

The bands at $480 \mathrm{~nm}(2.6 \mathrm{eV})$ observed in the PL spectra of the samples under study (Fig. 2); $500 \mathrm{~nm}(2.5 \mathrm{eV})$ characteristic of the luminescence of many silicates are usually associated with radiation, for example, defective centers $\left[\mathrm{SiO}_{4}\right]^{4-}$ caused by local distortions of silicon-oxygen tetrahedra [12]. Zirconium (zinc) ions can be in a tetrahedral environment in the form of $(\mathrm{OH})-\mathrm{Zr}-(\mathrm{OSi})_{3}$ or $\mathrm{Zr}-(\mathrm{OSi})_{4}$ groups and appear when the symmetry of the silicon-oxygen tetrahedron is broken due to a closely located defect.

Luminescence bands can also be associated with the creation of complex radiation defects: zinc, zirconium, oxygen vacancies, respectively, with the formation of anionic vacancies such as $\mathrm{F}$, $\mathrm{F}^{+}$as emission centers. The observed bands are possibly due to the intermediate stage of the formation of exciton-defect complexes or short-lived defects in the form of $\mathrm{V}_{\mathrm{Zr}^{-}}, \mathrm{V}_{\mathrm{Zn}^{-}}$vacancies and interstitial $\mathrm{Zr}^{+}, \mathrm{Zn}^{+}$ions formed upon bond breaking [3, 4]. Thus, it can be assumed that upon irradiation with electrons with energy of $4 \mathrm{MeV}$, the observed maxima in the PL spectra are due to the formation of point defects-anion vacancies.

TBCs subjected to heat treatment at $220{ }^{\circ} \mathrm{C}$ have an amorphous phase, which follows from the spectra of X-ray diffraction patterns. Therefore, the work presents TBC, which had a crystalline structure after heat treatment at $1050{ }^{\circ} \mathrm{C}$ and $1200{ }^{\circ} \mathrm{C}$.

The coefficient of thermal conductivity, which determines the magnitude of the heat flux, plays an important role in calculating the thermal regimes of various structures and mechanisms. However, there are no mathematical methods for calculating this coefficient; its value and dependence on temperature are determined experimentally.

In the steady-state measurement, the thermal conductivity and interfacial thermal conductance are determined by measuring the temperature difference $\Delta T$ at a separation (distance) under the steady-state heat flow $Q$ through the sample. Various stationary methods are commonly 
used: the absolute method, the comparative cut method of radial heat flow and the method of parallel heat conduction [13].

A variety of so-called "secondary" steady-state measurement techniques utilizing "comparison" of parameters of an unknown specimen with those of one or more specimens of known thermal properties exists. Here the fiberglass with thermal conductivity of $0.3 \mathrm{~W} /(\mathrm{m} \cdot \mathrm{K})$ was used as a standard material. The measurements were performed at room temperature $300 \mathrm{~K}$ and humidity $47 \%$.

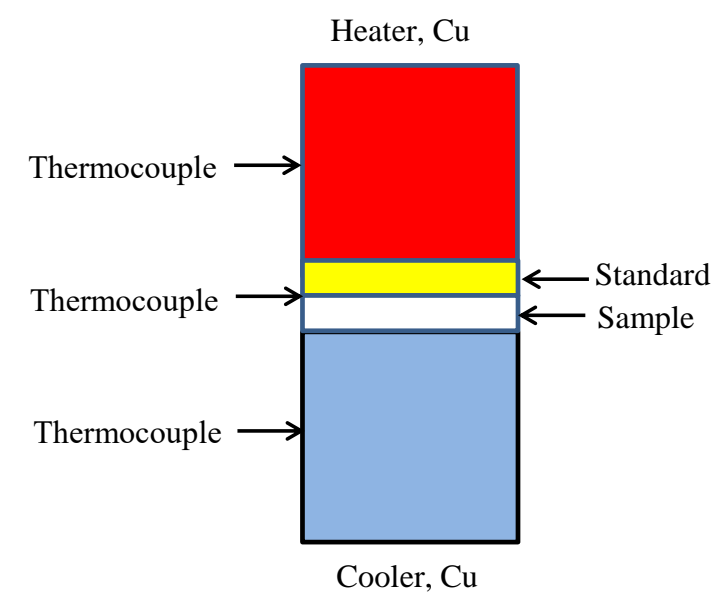

Fig. 3. Schematic comparative cut bar technique.

The thermal conductivity of the samples is determined by the following equation

$$
\lambda_{x}=\frac{\Delta \mathrm{X}}{\Delta \mathrm{X}_{0}} \frac{\mathrm{S}_{1}}{\mathrm{~S}_{2}} \frac{\Delta T_{21}}{\Delta T_{10}} \lambda_{0}
$$

where $\Delta X$ and $\Delta X_{0}$ are the thicknesses of the measured and standard samples, $\Delta T_{21}$ is the temperature difference between the contact surfaces of the samples and the heater, $\Delta T_{10}$ is the temperature difference between the contact surfaces of the samples and the cooler, $S_{1}$ and $S_{2}$ are the cross-sectional areas of the standard sample and the test sample, these areas are equal to each other $S_{1}=S_{2}, \lambda_{0}$ and $\lambda_{x}$ are the thermal conductivity coefficients of the standard and measured sample $\left(\lambda_{0}=0.3 \mathrm{~W} /(\mathrm{m} \cdot \mathrm{K})\right)$.

Since the temperature difference between the two surfaces of the samples does not exceed 10 ${ }^{0} C$, it can be assumed that the relationship between the thermocouple signal and the measured temperature in this range is linear, therefore the temperature ratio is equal to the ratio of the thermocouple signals.

As a result, we obtained a value of $0.07 \mathrm{~W} /(\mathrm{m} \cdot \mathrm{K})$ for the thermal conductivity of the sample $\mathrm{ZrSiO}_{4}\left(\mathrm{Ce}_{2} \mathrm{O}_{3}-5 \%\right)$ at a density of $1 \mathrm{~g} / \mathrm{cm}^{3}$, and $0.12 \mathrm{~W} /(\mathrm{m} \cdot \mathrm{K})$ for the sample of $\mathrm{Zn}_{2} \mathrm{SiO}_{4}\left(\mathrm{Ce}_{2} \mathrm{O}_{3-}-\right.$ $5 \%$ ) at the density of $2.4 \mathrm{~g} / \mathrm{cm}^{3}$. The obtained low values of thermal conductivity coefficients suggest that these samples can be used as pigment thermal barrier coatings.

\section{Conclusions}

Examining the radiative-optical properties of the samples, we can state that according to the results of X-ray diffraction analysis, the samples obtained by the hydrothermal microwave method at high temperatures have a crystalline structure. From the spectra of fluorescence, it can be seen 
that after electron irradiation, there is a slight increase in luminescence which suggests that new radiation centers are forming. The low values obtained for the coefficients of thermal conductivity allow us to assume that the coatings based on $\mathrm{Ce}-\mathrm{Zn}_{2} \mathrm{SiO}_{4}$, and $\mathrm{Ce}-\mathrm{ZrSiO}_{4}$ are excellent candidates for space vehicles.

\section{References}

[1] H.N. Yeritsyan, A.A. Sahakyan, S.K. Nikoghosyan, V.V. Harutunyan et al., J. Spacecr. Rockets 48 (2011) 34

[2] B.J. Lewis, A.R. Green, L.G.I. Bennett, Adv. Sp. Res. 44 (2009) 184.

[3] A.I. Akishin, Yu.V. Bulgakov, S.S. Vasiliev, S.N. Vernov, B.C. Nikolaev, I.B. Teplov, Modeling the radiation effect of the space environment on materials and equipment of space objects (M. NIIJa.F. MSU, 1968).

[4] M.M. Mikhailov, M.I. Dvoretsky, Geotechnics 3 (1981) 31.

[5] M.M. Mikhailov et al., Russian Physics Journal 6 (1998) 83.

[6] M. M. Mikhailov, Adv. Mater. 2 (1995) 200.

[7] V.V. Baghramyan, V.V. Harutyunyan, E.M. Aleksanyan, N.E. Grigoryan, A.H. Badalyan et al., Arm. J. Phys. 10 (2017) 56 .

[8] V.V. Baghramyan, A.A. Sargsyan, V.V. Harutyunyan, A.H. Badalyan, N.E. Grigoryan et al., Ceram. Int. 46 (2020) 4992.

[9] V.V. Bagramyan, A.A. Sarkisyan, C. Ponzoni, R. Rosa, C. Leonelli, Theor. Found. Chem. Eng. 49 (2015) 731.

[10] V. Harutyunyan et al., Arm. J. Phys. 9 (2016) 225.

[11] A.A. Sargsyan, V.V. Baghramyan, V.V. Harutyunyan, A.O. Badalyan, N.E. Grigoryan et al., J. Contemp. Phys. (Arm. Acad. Sci.) 55 (2020) 23.

[12] A.F. Zatsepin, A.I. Kukharenko, V.A. Pustovarov, V.Yu. Yakovleva, Solid State Phys. 51 (2009) 465.

[13] D. Zhao, X. Qian, X. Gu, S. A. Jajja, R. Yang, J. Electron. Packag. Trans. ASME 138 (2016) 1. 